\title{
Changes in dynamic transitions between integrated and segregated states underlie visual hallucinations in Parkinson's disease
}

Angeliki Zarkali ${ }^{1}$, Andrea I. Luppi ${ }^{2,3}$, Emmanuel A. Stamatakis ${ }^{2,3}$, Suzanne Reeves ${ }^{4}$, Peter McColgan $^{5}$, Louise-Ann Leyland ${ }^{1}$, Andrew J. Lees ${ }^{6}$, Rimona S. Weil ${ }^{1,7,8}$,

1. Dementia Research Centre, University College London, 8-11 Queen Square, London, WC1N 3AR

2. Division of Anaesthesia, School of Clinical Medicine, University of Cambridge, Cambridge CB2 0QQ, United Kingdom

3. Department of Clinical Neurosciences, University of Cambridge, Cambridge CB2 0QQ, United KingdomHuntington's Disease Centre, University College London, Russell Square House, London, WC1B 5EH

4. Division of Psychiatry, University College London, 149 Tottenham Court Rd, London W1T 7BN

5. Huntington's Disease Centre, University College London, Russell Square House, London, WC1B 5EH

6. Reta Lila Weston Institute of Neurological Studies, 1 Wakefield Street, London, WC1N 1PJ

7. Wellcome Centre for Human Neuroimaging, University College London, 12 Queen Square, London, WC1N 3AR

8. Movement Disorders Consortium, University College London, London WC1N 3BG

Keywords/Search terms: Parkinson's disease, visual hallucinations, dynamic functional connectivity, diffusion weighted imaging, regional gene expression, neurotransmitters, network control theory 


\section{Introduction}

Visual hallucinations are a common symptom of Parkinson's disease (PD) and are associated with cognitive decline(1), poorer quality of life(2) and increased mortality(3). The brain changes that give rise to hallucinations are not fully understood. However, the transient nature of hallucinations, even in patients who regularly experience them, suggests that the underlying process is a dynamic one and may be best examined using imaging techniques sensitive to dynamic changes in brain states. One potential approach is resting state functional MRI (rsfMRI), which measures spontaneous fluctuations in brain activity based on correlated fluctuations in blood oxygenation(4). This technique has shown changes in relative activity of specific functional brain networks in patients with PD-hallucinations(5), characterised by increased activation of the default mode network (DMN) and impaired recruitment of the dorsal attention network(6-9). However, these studies only provide a static image of functional connectivity, calculated over an entire scanning period, rather than examining dynamic changes in brain states.

An extension of this approach is dynamic functional connectivity analysis, which measures spontaneous fluctuations in connectivity over time(10-12) and may be a more accurate representation of fluctuating cognitive states than previous static approaches(13). Changes in temporal dynamics are seen in schizophrenia and other psychiatric conditions(14-17), and recent work showed imbalance of temporal dynamics of integrated and segregated states in anaesthesia and disorders of consciousness $(18,19)$ and after administration of the psychedelic LSD-25, known for its hallucinogenic properties(20). Changes in dynamic functional connectivity have been described in $\mathrm{PD}(21)$ and are associated with the severity of both motor and cognitive symptoms(22-24) but are as yet unexplored in relation to neuropsychiatric symptoms.

PD patients with hallucinations show widespread disruption in structural connections between brain regions, measured using diffusion $\operatorname{MRI}(25,26)$. These changes particularly affect highly connected brain regions or "hubs" important for switching the brain between different states $(27,28)$. This is important because structural connectivity constrains the temporal alteration between different brain states $(29,30)$ and the transitions between states can be modelled using network control theory(31). Specifically, the optimal energy cost needed to move the brain from one state to another can be calculated based on its 
structural network(31-33). A state that is less energy-demanding to maintain, or requires lower energy for transition, will be preferred. This framework can explain why a particular state is predominantly seen in health and how the balance between states may change in the presence of disease.

In this study we aimed to investigate the pattern of temporal dynamics in PD-associated visual hallucinations using rsfMRI; and determine whether the balance between integrated and segregated states is preserved in patients with PD and visual hallucinations compared to those without hallucinations and controls. We found that patients with Parkinson's hallucinations show impaired temporal dynamics, with a predisposition towards a segregated state. We then used network control theory to calculate each individual's required energy cost to transition from the integrated-to-the-segregated state and vice versa, and the cost to maintain each state. We found that patients with Parkinson's hallucinations required less energy to transition from the integrated-to-segregated state than those without hallucinations and controls. Finally, we identified the brain regions that contribute most to transitions from integrated-to-segregated state. As dynamic neural systems are, at least partly, modulated by neurotransmitter systems(34) we related the regional pattern of this transition to neurotransmitter systems using PET-derived regional neurotransmitter density profiles and regional gene expression for neurotransmitter receptors.

\section{Methods and Materials}

\section{Participants}

123 participants were included: 91 PD patients and 32 unaffected controls. All PD patients fulfilled Queen Square Brain Bank Criteria(35) and were recruited from clinics in the National Hospital for Neurology and Neurosurgery and affiliated hospitals. Controls were recruited from spouses and volunteer databases.

Patients with PD were classified as PD with visual hallucinations (PD-VH, $n=16$ ) if they scored $\geq 1$ in Question 2.1 of the Unified Parkinson's Disease Rating Scale (UPDRS). All other patients were classified as PD-non-VH ( $n=75)$. We collected additional information on 
bioRxiv preprint doi: https://doi.org/10.1101/2021.06.21.449237; this version posted June 21, 2021. The copyright holder for this preprint (which was not certified by peer review) is the author/funder, who has granted bioRxiv a license to display the preprint in perpetuity. It is made available under aCC-BY 4.0 International license.

severity, frequency and the phenomenology of experienced hallucinations with the University of Miami Parkinson's Disease Hallucinations Questionnaire (UM-PDHQ)(36).

Study participants underwent clinical assessments of general and specific cognition across 5 domains as well as PD-specific measures (Supplementary Methods 1).

A
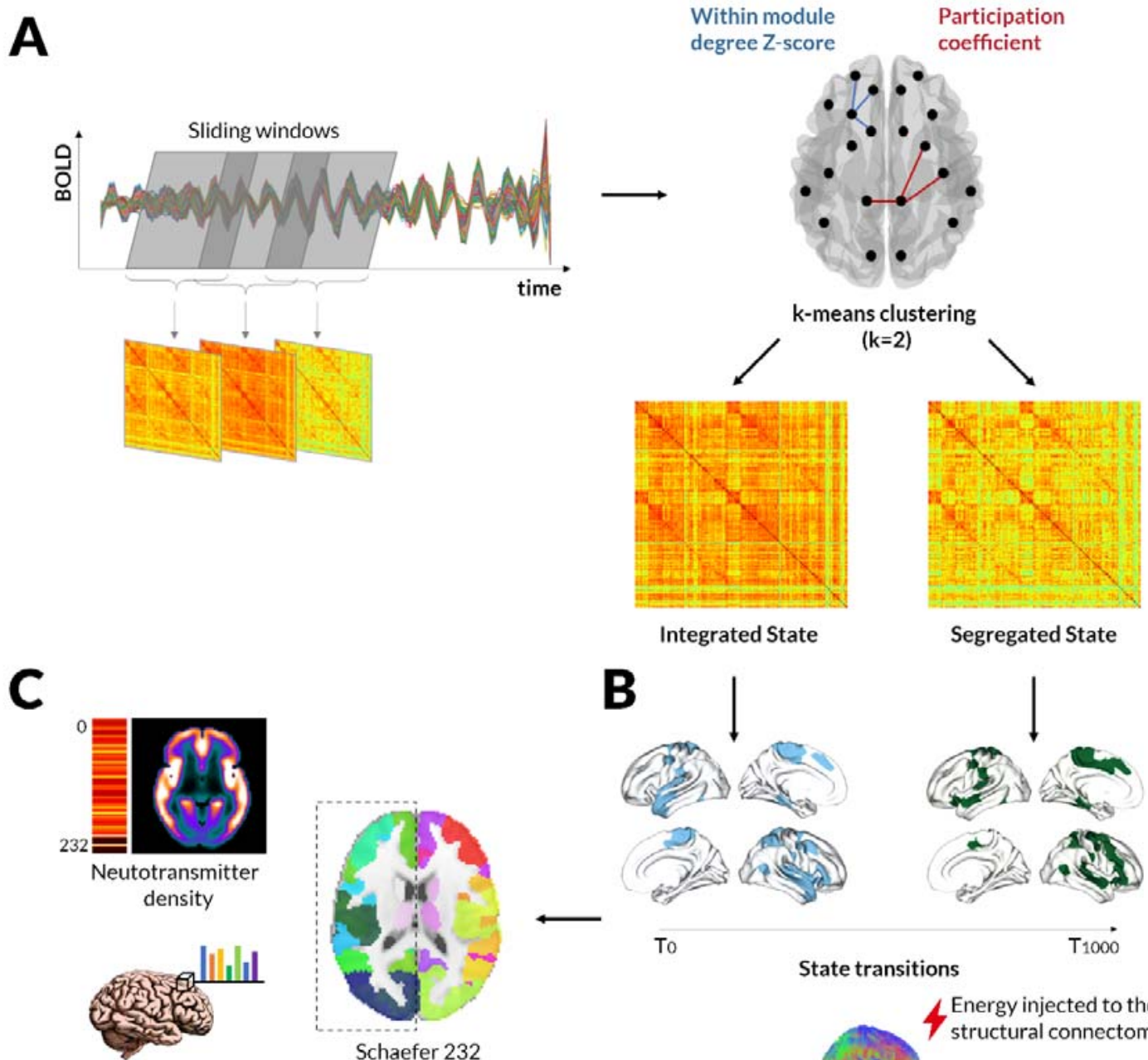

Segregated State

Regional gene

Schaefer 232 expression 
bioRxiv preprint doi: https://doi.org/10.1101/2021.06.21.449237; this version posted June 21, 2021. The copyright holder for this preprint (which was not certified by peer review) is the author/funder, who has granted bioRxiv a license to display the preprint in perpetuity. It is made available under aCC-BY 4.0 International license. 


\section{Figure 1. Overview of the study methodology}

\section{A. Deriving integrated and segregated states of dynamic functional connectivity.}

After obtaining sliding-windows (each 44s duration) of dynamic functional connectivity for each participant, the joint histogram of participation coefficient and within-module degree Z-score was used for $k$-means clustering $(k=2)$. (BOLD, blood oxygen level dependent activity).

The cluster with highest average participation coefficient is then identified as the predominantly Integrated dynamic state and the cluster with the lowest participation coefficient as the predominantly Segregated state. Note that this is done for each participant separately leading to individually-defined Integrated and Segregated states.

\section{B. Modelling state transitions.}

After deriving each individual's Integrated and Segregated states we used an optical control framework to calculate the minimal control energy that needs to be applied to each node of the structural network to transition from a baseline state at time $T_{0}$ to a target state at time $\mathrm{T}_{1000}$. Here, as an example, we illustrate the transition from the Integrated state (top $20 \%$ of nodes in blue) to the Segregated state (top $20 \%$ of nodes in green) but minimal energies were also calculated for Segregated-to-Integrated transition as well as minimal energies to maintain the integrated state (Integrated-to-integrated) and segregated state (segregatedto-segregated) using the same model.

Minimal control energies were calculated for each subject based on their structural brain network, which was estimated using diffusion imaging and probabilistic tractography. Both states were represented in the model as a vector of the sum connectivity strength for each node $(1 * 232)$.

\section{Linking with neurotransmitter systems.}

Minimal control energies to transition between and maintain functional states were compared between patients with PD with (PD-VH) and without hallucinations (PD-non-VH). Transitions that differed between groups were then further explored to examine whether contributing nodes (requiring mode control energy) were associated with specific neurotransmitter systems. To do this, we calculated for each of the 232 regions of interest of our parcellation (Schaeffer 232: 200 cortical and 32 subcortical regions) 1) mean neurotransmitter density profiles derived from PET data (serotonin (5HT1a, 5HT2 $a$ and $5 H T 1 b$ ), dopamine ( $D 1$ and $D 2$ ) and $G A B A_{A}$ receptors) and 2) gene expression profiles for each of 31 pre-selected genes encoding receptors for norepinephrine, acetylcholine, dopamine and serotonin. 


\section{Results}

Study participants included 16 PD patients with habitual visual hallucinations (PD-VH), 75 PD patients without hallucinations (PD-non-VH) and 32 controls. PD-VH and PD-non-VH were well matched in demographics, cognitive and motor performance, levodopa equivalent dose, and image quality (Supplementary Table 1).

\section{Preserved topology of dynamic functional connectivity states}

To examine the dynamic changes in functional connectivity underlying PD-hallucinations, we employed an a-priori clustering of dynamic functional connectivity into two states, an Integrated and a Segregated state. We found no significant differences within each state between PD versus controls or PD-VH versus PD-non-VH when comparing connectivity strength in each state using network-based statistics. We also found no significant betweengroup differences in the Integrated and Segregated states in terms of connectivity density (Integrated: Kruskal Wallis $\mathrm{H}=2.473, \mathrm{p}=0.290$, Segregated: $0.175, \mathrm{p}=0.529$ ), entropy of connectivity values (Integrated: $\mathrm{H}=0.723, \mathrm{p}=0.696$, Segregated: $\mathrm{H}=0.905, \mathrm{p}=0.636$ ), structural-functional coupling (Integrated $\mathrm{F}(111,2)=1.093, \mathrm{p}=0.339$, Segregated: $F(111,2)=1.401, p=0.251$ ) or small world propensity (Integrated: $\mathrm{H}=1.065, \mathrm{p}=0.587$, Segregated: $\mathrm{H}=4.400, \mathrm{p}=0.111$ ).

\section{Impaired temporal properties of dynamic functional connectivity in Parkinson's hallucinations}

Although the states themselves were preserved between groups in terms of network properties, we found significant changes in their temporal properties. PD-VH spent a significantly smaller proportion of time in the Integrated state (therefore higher proportion of time in the Segregated state) than PD-non- $\mathrm{VH}(\beta=-0.113, \mathrm{p}=0.032)$ and than controls ( $\beta=-$ $0.128, \mathrm{p}=0.026$ ) (Figure 2A). Within PD patients, the proportion of time spent in the Integrated state was inversely correlated with hallucination severity, measured by the UMPDHQ ( $\rho=-0.259, p=0.013$ ) (Figure 2B). Mean dwell time in the Segregated state was higher in PD-VH than PD-non-VH (19.1 \pm 16.9 in PD-VH vs $9.5 \pm 9.1$ in PD-non-VH H=4.058, p=0.044), with no difference between the two groups in mean dwell time of the Integrated state $(H=2.166, p=0.141)$. No differences were seen in dwell time of either state between PD and controls. Finally, the total number of transitions was lower in PD-VH than PD-non-VH 
(5.7 \pm 5.3 in PD-VH vs $8.5 \pm 6.2$ in PD-non-VH, $H=3.87, p=0.049$ ) but there was no difference between the two groups when the number of transitions from integrated-to-segregated state and segregated-to-integrated state were examined separately.

Overall, this suggests that PD-VH spend more time in the Segregated state than PD-non-VH, with fewer total transitions and longer dwelling time within the Segregated state (Figure 2).

\section{Figure 2. Altered temporal properties of dynamic functional connectivity in patients with Parkinson's and visual hallucinations}

\section{A. Percentage of total time spent in the Integrated state.}

Patients with Parkinson's with visual hallucinations spent significantly less time in the Integrated state of dynamic functional connectivity than patients without hallucinations $(p=0.032)$ and controls $(p=0.0262)$. (Error bars are $95 \%$ confidence intervals)

\section{B. Correlation between proportion of time spent in the Integrated state and hallucination severity}

The proportion of time spent in the integrated State was significantly correlated with hallucination severity in participants with Parkinson's disease (Spearman's correlation coefficient $\rho=-0.259, p=0.013$ ): participants with more severe hallucinations spent less time in the integrated state.

PD-VH: Parkinson's disease with visual hallucinations, PD-non-VH: Parkinson's disease without hallucinations. UM-PDHQ: University of Miami Parkinson's disease Hallucinations Questionnaire, higher scores indicate more severe and frequent hallucinations.
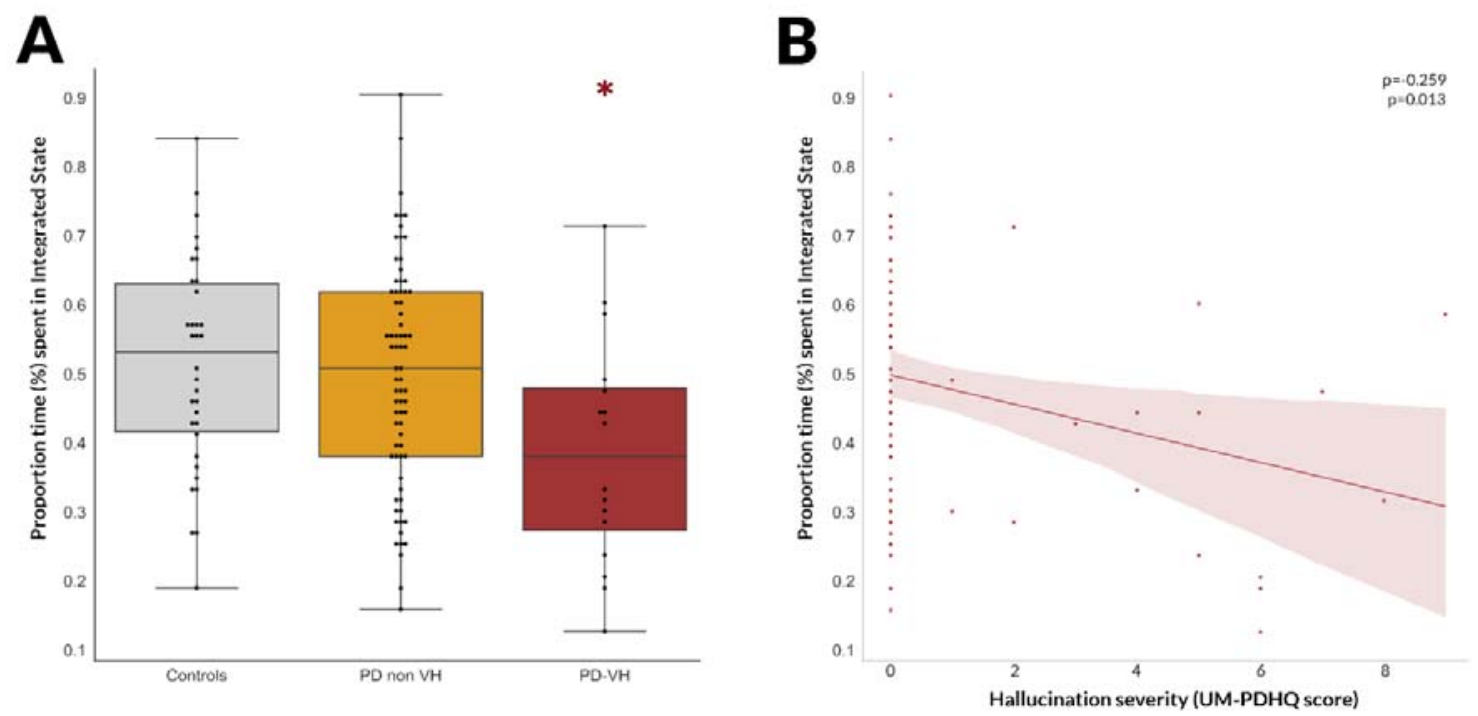


\section{Reduced energy costs to transition from the integrated to segregated state in Parkinson's patients with visual hallucinations}

Having identified significant differences in terms of brain dynamics between PD-VH and PDnon- $\mathrm{VH}$, which are specifically related to the severity of visual hallucinations (the focus of our present investigation), we sought to interrogate further this difference between PD patients. Specifically, we aimed to investigate whether the Segregated state predominance observed in PD-VH participants could be explained by differences in ease of transition from the integrated-to-segregated state or vice versa or a difference in ease of maintaining the Segregated state in PD-VH compared to PD-non-VH participants. To do this, we calculated the minimal control energy that needs to be applied to the structural network of each participant to 1) transition from integrated-to-segregated state 2) transition from segregated-to-integrated state 3) maintain the integrated state and 4) maintain the segregated state. We then examined whether transition and persistence energies in each state differed between PD-VH and PD-non-VH.

Similarly to previous work(64), persistence energy for the computationally more demanding Integrated state was higher than the Segregated state for all participants (log(Persistence Energy) Integrated: $13.8 \pm 0.9$ vs $13.7 \pm 1.1$ Segregated, repeated measures ANOVA main effect of Integrated to Segregated state persistence energy $F(1,113)=12.432, p<0.001)$. Similarly the minimal energy needed to transition from the less connected Segregated to the more interconnected Integrated state was higher (Integrated-to-Segregated $13.9 \pm 0.7$ vs Segregated-to-Integrated 14.1 $\pm 0.8, \mathrm{~F}(1,113)=6.722, \mathrm{p}=0.011$ ) (Supplementary Figure 2).

PD-VH needed significantly lower control energy to transition from the integrated-tosegregated state than PD-non-VH (effect size Hedge's $g=0.922, t=2.376, p=0.029$ ) (Figure $3 A)$. The minimal control energy to transition from an Integrated-to-Segregated state was significantly correlated with hallucinations severity: lower energy associated with more severe hallucinations $(\rho=-0.283, p=0.008)$ (Figure $3 B$ ). There were no statistically significant differences between PD-VH and PD-non-VH in the minimal control energy needed to transition from segregated-to-integrated state $(t=1.346, p=0.195)$, or to persist within the Integrated $(\mathrm{t}=1.041, \mathrm{p}=0.312)$ or Segregated state $(\mathrm{t}=1.079, \mathrm{p}=0.295)$. Therefore, network control theory reveals that the higher proportion of time that PD-VH patients spend in the 
Segregated state may be accounted for in terms of this state being easier to transition to from the Integrated state (as opposed to being easier to persist in).

\section{Figure 3. Changes in control energy to transition from the Integrated to the Segregated state in patients with Parkinson's and visual hallucinations}

\section{A. Minimal control energy to transition from the Integrated to the Segregated state}

Less energy is needed to transition for patients with Parkinson's and visual hallucinations (PD-VH) than those without hallucinations (PD-non-VH). Log-transformed minimal control energy is presented. Error bars are $95 \%$ confidence intervals

\section{B. Correlation between minimal control energy and hallucination severity}

The log-transformed minimal control energy required across the whole of the network to transition from the Integrated to the Segregated state was significantly correlated with severity in participants with Parkinson's disease (Spearman's correlation coefficient $\rho=-$ $0.283, p=0.008$ ): participants with more severe hallucinations needed less energy to transition, suggesting that the transition to the Segregated state may be easier to achieve in PD-VH therefore prefered.

\section{Regional variation in minimal control energy to transition from the Integrated to the Segregated state}

The log-transformed minimal control energy that needs to be applied to each node is presented; darker colours denote higher amounts of energy required. Note that only cortical regions are plotted.

\section{Minimal control energy per functional subnetwork.}

The mean minimal control energy to transition from the Integrated to the Segregated state across all nodes of the seven cortical and one subcortical resting state networks is plotted. Darker colours denote higher levels of the cortical hierarchy; also left to right: unimodal to transmodal regions. There was a significant correlation between the minimal transition energy from integrated-to-segregated state that was needed to be applied to each node and the nodes position in the cortical hierarchy, with higher amount of energy needed for more transmodal regions $(\rho=0.526, p<0.001)$. 
bioRxiv preprint doi: https://doi.org/10.1101/2021.06.21.449237; this version posted June 21, 2021. The copyright holder for this preprint (which was not certified by peer review) is the author/funder, who has granted bioRxiv a license to display the preprint in perpetuity. It is made available under aCC-BY 4.0 International license. 
bioRxiv preprint doi: https://doi.org/10.1101/2021.06.21.449237; this version posted June 21, 2021. The copyright holder for this preprint (which was not certified by peer review) is the author/funder, who has granted bioRxiv a license to display the preprint in perpetuity. It is made available under aCC-BY 4.0 International license.

A

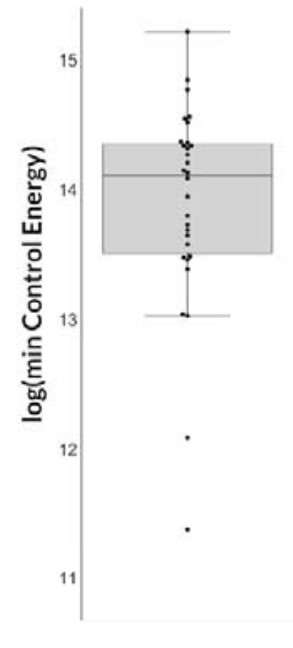

Controls

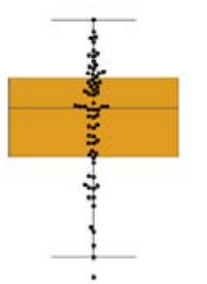

•

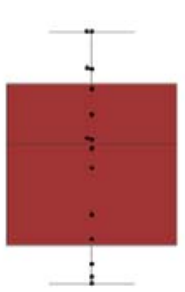

B

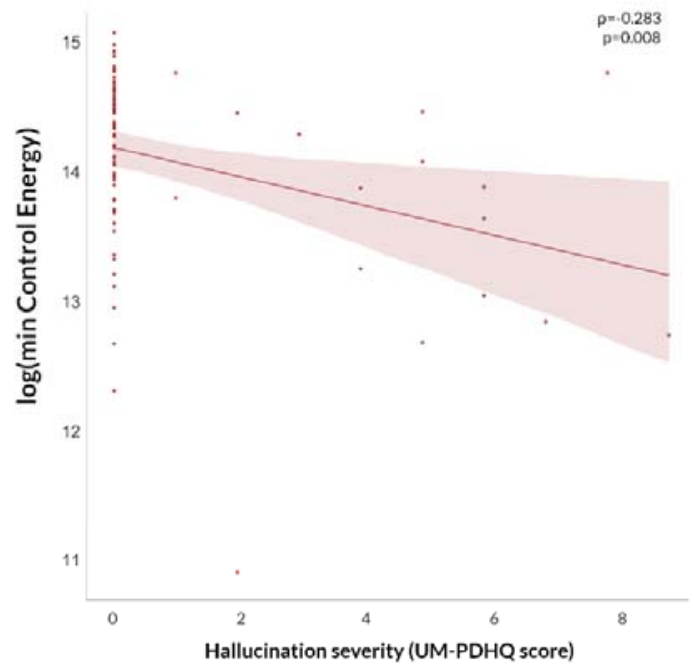

C
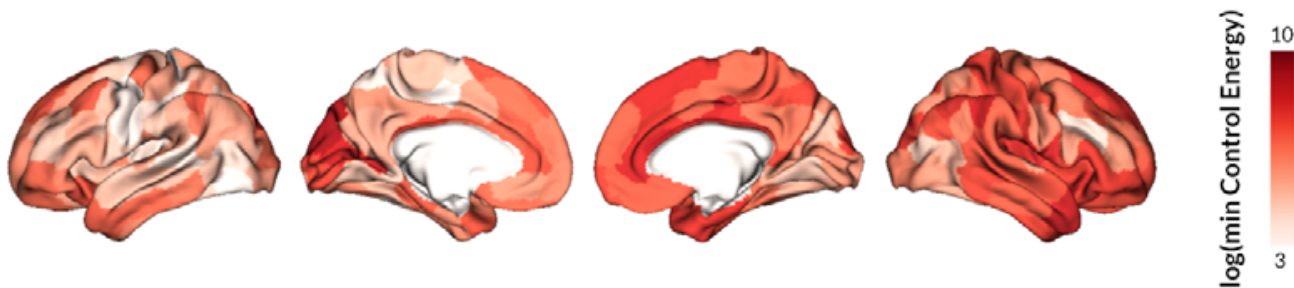

D

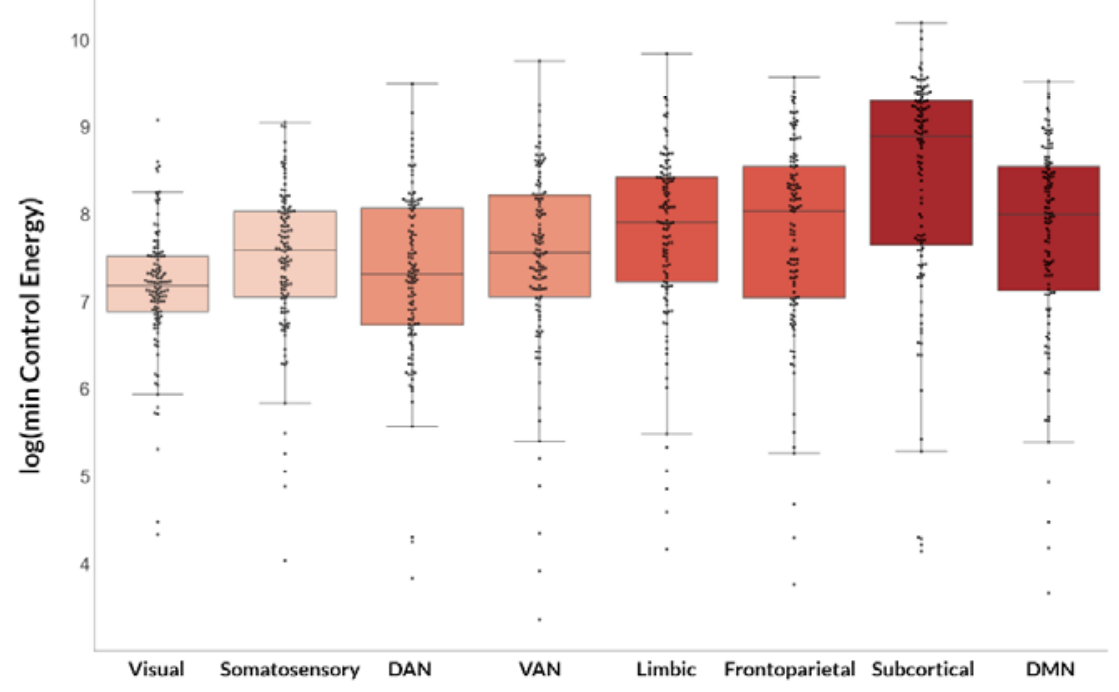




\section{Transition from integrated to the segregated state is driven by subcortical and more multimodal brain regions}

Next, we aimed to identify which brain regions contribute more to the transition from the Integrated-to-Segregated state (which nodes need more energy to be applied to transition). As expected(65), subcortical regions heavily contributed, with 25 subcortical nodes amongst the top $20 \%$ of contributors (25/47 or $53.2 \%$ ) with thalamic regions amongst the highest contributors. Amongst cortical nodes, top contributors included primarily right hemispheric regions (20/22 cortical nodes) including regions of the Default mode network: cingulum, precuneus, inferior and superior temporal regions and medial frontal regions (Figure 3C). There was a significant correlation between the transition energy from Integrated-toSegregated state that needed to be applied to each node and the node's position in the cortical hierarchy, with higher energy needed for more transmodal regions $(\rho=0.526$, $\mathrm{p}<0.001$ ) (Figure 3D for regional energy per functional network).

\section{Correlation with neurotransmitter systems}

Finally, we examined whether the Integrated-to-Segregated state transition, which was less costly for PD-VH patients is associated with specific neurotransmitter systems in the healthy brain. To do this, we correlated the mean control per node to transition from the Integrated-to-Segregated state with mean regional neurotransmitter density (derived from open-access PET data) and neurotransmitter receptor gene expression levels (derived from the Allen Brain atlas(61)) in health; we tested this against spatially-correlated null models through sphere permutations, FDR-corrected for multiple comparisons.

We found a significant correlation between regional $\log ($ Energy) and density of $5-H T 1 b$ ( $\rho=-$ $\left.0.274, q_{\text {spin }}=0.009\right), 5-H T 2 a\left(\rho=-0.347, q_{\text {spin }}<0.001\right)$ and $G A B A_{A}$ receptors $(\rho=-0.317$, $q_{\text {spin }}=0.022$ ), from open-access atlases of PET data. Regional energy and regional expression levels of genes relating to 5 -HT2a receptors were also significantly correlated $(\rho=-0.1438$, $\left.q_{\text {spin }}=0.044\right)$ as well as two $G_{A B A}$ receptors [GABRA1 $\left(\rho=-0.2437, q_{\text {spin }}=0.020\right)$ and $G A B R A 2$ $\left.\left(\rho=0.128, q_{\text {spin }}=0.023\right)\right]$; gene expression data for $5-H T 1 b$ receptors were not available. Although noradrenergic and acetylcholinergic PET data are not publically available, genetic expression of noradrenergic (ADRA1B and ADRA2A), muscarinic (CHRM1, CHRM2, CHRM3, CHRM4) and nicotinic receptors (CHRNA3, CHRNA4, CHRNA7, CHRNB2) was correlated with 
regional transition energy. Gene expression of DRD2 was also correlated with regional control energy for the Integrated-to-Segregated state transition $\left(\rho=0.318, q_{\text {spin }}=0.013\right)$ but this was not replicated using density PET-derived data $\left(\rho=0.056, q_{\text {spin }}=0.800\right)$.

\section{Figure 4. Neurotransmitter correlates of Integrated-to-Segregated state transition.}

The log-transformed minimal control energy that needs to be applied to each node (A) was correlated with the mean regional receptor density of $5 \mathrm{HT} 1 \mathrm{~b}$ receptors (B), $5 \mathrm{HT} 2 \mathrm{a}$ receptors (C) and GABA receptors (D), from open access atlases of PET data in unaffected individuals. In all cases $\rho$ is the Spearman correlation coefficient and $q$-spin is the FDR corrected $p$-value derived following spatial permutations ( $p$-spin, 1000 permutations).

A
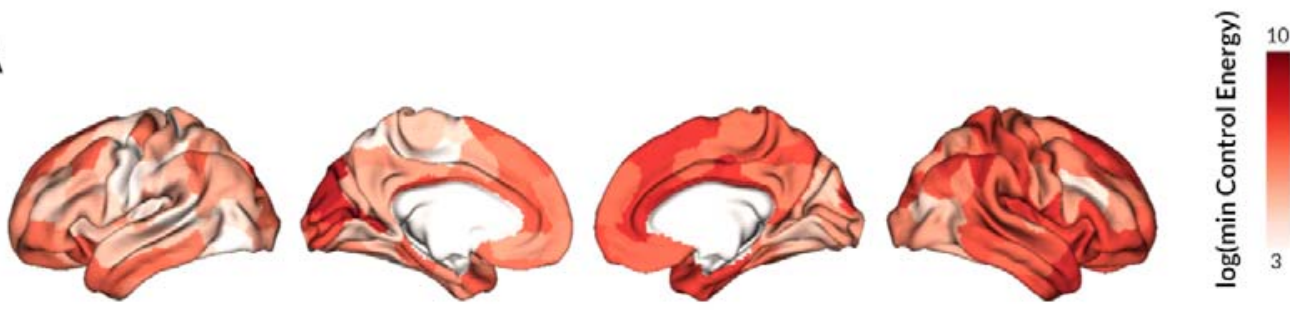

B

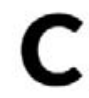

D
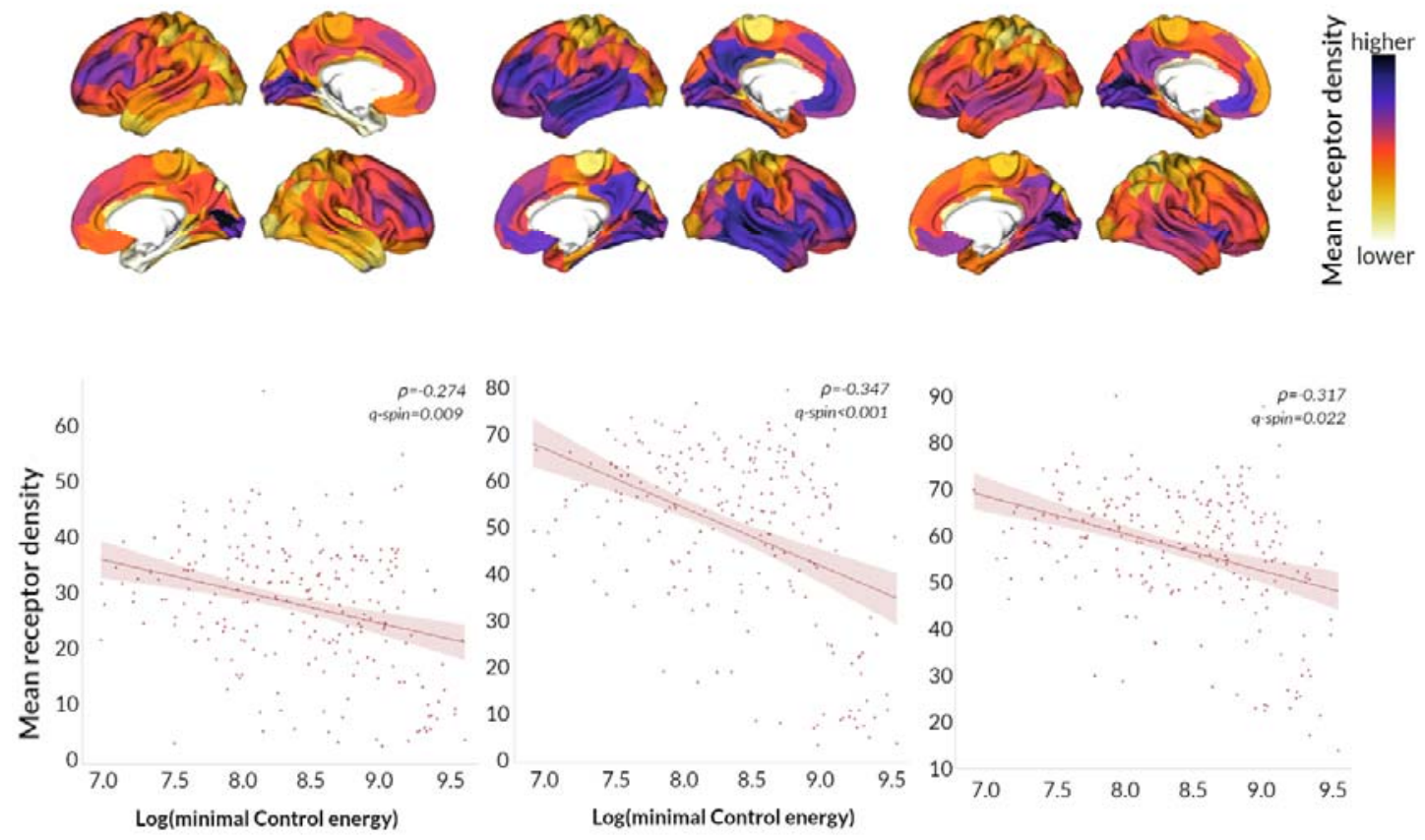

$5 \mathrm{HT} 1 \mathrm{~b}$ 


\section{Discussion}

We have used dynamic functional connectivity and network control theory to explore the temporal dynamics underlying visual hallucinations in Parkinson's, and examine how these specific patterns of temporal dynamics can be explained through brain structure. We found that PD-hallucinators spent more time in a Segregated state of functional connectivity than those without hallucinations, with fewer total transitions and longer dwelling time within the Segregated state. The transition from the Integrated-to-Segregated state was less energy demanding in PD-hallucinators than non-hallucinators. This transition is mediated by trans-modal brain regions that are associated with specific neurotransmitter systems, as confirmed through combined in-vivo PET mapping and post-mortem gene expression microarray data.

Previous studies have shown that PD patients with cognitive impairment similarly spend more time in a segregated state and show fewer transitions between states than PD with intact cognition and controls $(23,24)$. There were no differences in cognitive performance between PD patients with and without hallucinations in our cohort, but visual hallucinations are known to be associated with incipient dementia in PD(66).

In schizophrenia, where auditory hallucinations are a core feature, similar findings of altered dwell time are seen $(14,67)$, correlated with severity of hallucinations(68). We similarly saw a correlation with hallucination severity with patients with more severe visual hallucinations spending less time in the Integrated (and more time in the Segregated) state suggesting this finding is specific to hallucinations as a trait.

Only the temporal dynamics of functional connectivity were altered in PD with hallucinations. This indicates that a change in the temporal balance between normal/preserved states rather than a change in the states themselves underlie PDhallucinations. Using similar methodologies, studies in loss of consciousness and after LSD25 ingestion in healthy volunteers show within-state changes particularly within the Integrated state $(18,20)$, which is more linked to cognitive performance and alertness(12). As we examined the propensity to hallucinate rather than the hallucinatory state (participants were not actively experiencing hallucinations during scanning) it is possible that additional within-state changes could underlie visual hallucinations in PD, in the moment when they 
actually occur, an avenue for potential future investigations. In addition, although hallucinations in our participants were frequent (at least weekly) they were not universally complex and severe. In contrast, the acute LSD-induced visual hallucinations are believed to be due to serotoninergic system activation alone (specifically the $2 \mathrm{~A}$ receptor(69)), and are associated with changes in other sensory modalities including time/space dysperceptions and ego dissolution(70), which are not seen with PD-associated hallucinations; thus it is not unexpected that the underlying changes in temporal dynamics are different.

As temporal transition between functional states is constrained by structural connectivity $(29,30,32)$, we examined the energy cost of transitioning between and maintaining the Integrated and Segregated states. There was a significantly lower energy cost to transition from the Integrated-to-Segregated state for PD-hallucinators than nonhallucinators, suggesting that this transition is easier to achieve in hallucinators. This transition is mediated primarily by subcortical and multimodal brain regions of DMN, further highlighting its involvement in PD hallucinations(6). Thalamic regions were amongst the highest contributors to this transition. Thalamic involvement has been previously described in visual hallucinations $(56,71)$ and we recently showed longitudinal changes in grey and white matter within the medial mediodorsal thalamus(72). This provides further evidence of the thalamus as a key driver of network imbalance in PD-hallucinations $(65,73)$.

Interestingly, the brain regions contributing most to this transition from Integrated-toSegregated state showed a correlation with specific neurotransmitter systems in health. Although the directionality of the relationship is difficult to interpret as data on regional neurotransmitter density and gene expression were derived from healthy individuals, regional density of $5 H T 2 A$ receptors was significantly correlated with the regional control energy needed for Integrated-to-Segregated state transition; this was replicated using regional expression data for the $5 H T 2 A$ receptor gene.

Activation of $5 \mathrm{HT} 2 \mathrm{~A}$ receptors is a key mechanism for drug-induced hallucinations occurring with the psychedelic drugs, LSD-25, psilocybin and ayahuasca(74) and modelling studies have shown that this receptor plays a key role in engendering the characteristic brain dynamics of LSD(69). 5HT2A has also been implicated in PD-hallucinations; evidenced by the higher density of 5HT2A receptors within frontal, temporal and occipital regions in patients 
with PD hallucinations in post mortem and in vivo studies $(75,76)$ and the efficacy of the novel 5HT2A inverse agonist Pimavanserin in the treatment of PD-hallucinations(77).

Other serotonergic receptors were also important for the Integrated-to-Segregated state transition including: 5HT1B (receptor density, no genetic expression data), 5HT1E, 5HT1F and 5HT5A (gene expression data only). The correlation with multiple serotonin receptors, indicates that serotonergic modulators targeting multiple receptors could be potential therapeutic targets for PD-hallucinations. Of note, no receptor density or gene expression data were available for $5 \mathrm{HT} 3$ receptors, a target of interest for Ondansetron, 5HT3antagonist currently under evaluation as a treatment of hallucinations(78).

Regional receptor density and gene expression for GABAergic receptors were also correlated with regional transition energy in line with previous studies showing reduced GABA concentration in the visual cortex of PD-hallucinators $(79,80)$. Visual processing involves a complex interplay between monoaminergic, cholinergic and GABA/glutamatergic neurotransmission(73). The observed correlation between the Integrated-to-Segregated state transition and regional gene expression of noradrenergic (ADRA1B, ADRA2A) and cholinergic (muscarinic and nicotinic) receptors is consistent with this, but there was no available PET derived density data to replicate this.

Convergent evidence has recently highlighted the importance of the noradrenergic system in some non-motor PD symptoms(81-83). Noradrenaline plays a key role in modulating selective attention(84) and with serotonin, modulates behavioural responses to incoming visual information(73). Changes within the noradrenergic system may be involved in altered state transitions in PD-hallucinations by modulating the activity of sensory cortices and thalamocortical neurocircuitry(85). In contrast there was no consistent correlation with dopaminergic receptors. These findings highlight the role of transmitters other than dopamine in the development of PD-hallucinations.

Several considerations need to be taken into account when interpreting our findings. Functional data are susceptible to motion artefact; we adopted strict exclusion criteria to mitigate for this(86). Global signal regression is a potential additional tool to counteract residual artifacts from head motion(86) however it may contain behaviourally-relevant information and affect group results $(40,87)$, therefore we did not regress global signal 
$(18,20)$. All participants were scanned while receiving their usual dopaminergic medications and at the same time of day and levodopa equivalent doses did not significantly differ between PD-VH and PD-non-VH(88). Further studies assessing PD patients ON and OFF levodopa might provide additional information. Although brain networks are non-linear, we used a linear optimal control model since this has been shown to provide important insights on non-linear dynamics(89) and linear-Gaussian models are often adequate descriptors of functional MRI timeseries, such that more complex, non-linear models often do not provide additional explanatory power $(90,91)$. Nevertheless, future work may seek to leverage insights from non-linear models of brain dynamics, e.g. through neurobiologically detailed dynamic mean-field models that have already been successfully applied to the study of altered states of consciousness $(69,92)$. Finally, data on neurotransmitter density and gene expression were not derived from our participants but from separate cohorts of healthy volunteers and post-mortem human brains respectively; therefore results relating to neurotransmitter receptors should be interpreted with caution. Future work may seek to replicate these results with each patient's own unique neurotransmitter receptor signature, which may offer individualised insights and the opportunity to assess the directionality of this relationship, as well as potential targets for pharmacological intervention.

\section{Conclusions}

Our findings describe, for the first time, that temporal functional dynamics are altered in PD-hallucinations, with a predisposition towards a segregated state of functional connectivity. This segregated state predominance can be explained by a reduced energy cost to transition from the integrated-to-segregated state in PD patients with hallucinations compared to those without hallucinations. We have also clarified the neuromodulatory correlates of the integrated-to-segregated state transition in the healthy brain. These results provide mechanistic insights into visual hallucinations in PD and possible therapeutic targets. 


\section{References}

1. Hobson P, Meara J (2004): Risk and incidence of dementia in a cohort of older subjects with Parkinson's disease in the United Kingdom. Mov Disord Off J Mov Disord Soc 19: 1043-1049.

2. McKinlay A, Grace RC, Dalrymple-Alford JC, Anderson T, Fink J, Roger D (2007): A Profile of Neuropsychiatric Problems and Their Relationship to Quality of Life for Parkinson's Disease Patients Without Dementia. Park Relat Disord 14 (1): 37-42.

3. Goetz CG, Stebbins GT (1995): Mortality and hallucinations in nursing home patients with advanced Parkinson's disease. Neurology 45: 669-671.

4. Fox MD, Raichle ME (2007): Spontaneous fluctuations in brain activity observed with functional magnetic resonance imaging. Nat Rev Neurosci 8: 700-711.

5. Muller AJ, Shine JM, Halliday GM, Lewis SJG (2014): Visual hallucinations in Parkinson's disease: theoretical models. Mov Disord Off J Mov Disord Soc 29: 1591-1598.

6. Shine JM, Halliday GM, Gilat M, Matar E, Bolitho SJ, Carlos M, et al. (2014): The role of dysfunctional attentional control networks in visual misperceptions in Parkinson's disease. Hum Brain Mapp 35: 2206-2219.

7. Shine JM, Keogh R, O'Callaghan C, Muller AJ, Lewis SJG, Pearson J (2015): Imagine that: elevated sensory strength of mental imagery in individuals with Parkinson's disease and visual hallucinations. Proc Biol Sci 282: 20142047.

8. Yao N, Shek-Kwan Chang R, Cheung C, Pang S, Lau KK, Suckling J, et al. (2014): The default mode network is disrupted in Parkinson's disease with visual hallucinations. Hum Brain Mapp 35: 5658-5666.

9. Baggio HC, Segura B, Junque C (2015): Resting-state functional brain networks in Parkinson's disease. CNS Neurosci Ther 21: 793-801. 
10. Allen EA, Damaraju E, Plis SM, Erhardt EB, Eichele T, Calhoun VD (2014): Tracking WholeBrain Connectivity Dynamics in the Resting State. Cereb Cortex 24: 663-676.

11. Hutchison RM, Womelsdorf T, Allen EA, Bandettini PA, Calhoun VD, Corbetta M, et al. (2013): Dynamic functional connectivity: promise, issues, and interpretations.

Neurolmage 80: 360-378.

12. Shine JM, Bissett PG, Bell PT, Koyejo O, Balsters JH, Gorgolewski KJ, et al. (2016): The Dynamics of Functional Brain Networks: Integrated Network States during Cognitive Task Performance. Neuron 92: 544-554.

13. Liégeois R, Li J, Kong R, Orban C, Van De Ville D, Ge T, et al. (2019): Resting brain dynamics at different timescales capture distinct aspects of human behavior. Nat Commun 10: 2317.

14. Sakoğlu U, Pearlson GD, Kiehl KA, Wang YM, Michael AM, Calhoun VD (2010): A method for evaluating dynamic functional network connectivity and task-modulation: application to schizophrenia. Magma N Y N 23: 351-366.

15. Kaiser RH, Whitfield-Gabrieli S, Dillon DG, Goer F, Beltzer M, Minkel J, et al. (2016):

Dynamic Resting-State Functional Connectivity in Major Depression. Neuropsychopharmacol Off Publ Am Coll Neuropsychopharmacol 41: 1822-1830.

16. Jin C, Jia H, Lanka P, Rangaprakash D, Li L, Liu T, et al. (2017): Dynamic brain connectivity is a better predictor of PTSD than static connectivity. Hum Brain Mapp 38: 44794496.

17. Rashid B, Arbabshirani MR, Damaraju E, Cetin MS, Miller R, Pearlson GD, Calhoun VD (2016): Classification of schizophrenia and bipolar patients using static and dynamic resting-state fMRI brain connectivity. Neurolmage 134: 645-657. 
18. Luppi Al, Craig MM, Pappas I, Finoia P, Williams GB, Allanson J, et al. (2019):

Consciousness-specific dynamic interactions of brain integration and functional diversity [no. 1]. Nat Commun 10: 4616.

19. Luppi Al, Golkowski D, Ranft A, Ilg R, Jordan D, Menon DK, Stamatakis EA (2021): Brain network integration dynamics are associated with loss and recovery of consciousness induced by sevoflurane. Hum Brain Mapp 42: 2802-2822.

20. Luppi Al, Carhart-Harris RL, Roseman L, Pappas I, Menon DK, Stamatakis EA (2021): LSD alters dynamic integration and segregation in the human brain. Neurolmage 227: 117653.

21. Cordes D, Zhuang X, Kaleem M, Sreenivasan K, Yang Z, Mishra V, et al. (2018): Advances in functional magnetic resonance imaging data analysis methods using Empirical Mode Decomposition to investigate temporal changes in early Parkinson's disease. Alzheimers Dement Transl Res Clin Interv 4: 372-386.

22. Kim J, Criaud M, Cho SS, Díez-Cirarda M, Mihaescu A, Coakeley S, et al. (2017): Abnormal intrinsic brain functional network dynamics in Parkinson's disease. Brain J Neurol 140: 2955-2967.

23. Díez-Cirarda M, Strafella AP, Kim J, Peña J, Ojeda N, Cabrera-Zubizarreta A, IbarretxeBilbao N (2018): Dynamic functional connectivity in Parkinson's disease patients with mild cognitive impairment and normal cognition. Neurolmage Clin 17: 847-855.

24. Fiorenzato E, Strafella AP, Kim J, Schifano R, Weis L, Antonini A, Biundo R (2019):

Dynamic functional connectivity changes associated with dementia in Parkinson's disease. Brain 142: 2860-2872. 
25. Zarkali A, McColgan P, Leyland L-A, Lees AJ, Rees G, Weil RS (2020): Fiber-specific white matter reductions in Parkinson hallucinations and visual dysfunction. Neurology. https://doi.org/10.1212/WNL.0000000000009014

26. Rau Y-A, Wang S-M, Tournier J-D, Lin S-H, Lu C-S, Weng Y-H, et al. (2019): A longitudinal fixel-based analysis of white matter alterations in patients with Parkinson's disease. Neurolmage Clin 24. https://doi.org/10.1016/j.nicl.2019.102098

27. Hall JM, O'Callaghan C, Muller AJ, Ehgoetz Martens KA, Phillips JR, Moustafa AA, et al. (2019): Changes in structural network topology correlate with severity of hallucinatory behavior in Parkinson's disease. Netw Neurosci 3: 521-538.

28. Zarkali A, McColgan P, Ryten M, Reynolds R, Leyland L-A, Lees AJ, et al. (2020):

Differences in network controllability and regional gene expression underlie hallucinations in Parkinson's disease. Brain 143: 3435-3448.

29. Stiso J, Khambhati AN, Menara T, Kahn AE, Stein JM, Das SR, et al. (2019): White Matter Network Architecture Guides Direct Electrical Stimulation through Optimal State Transitions. Cell Rep 28: 2554-2566.e7.

30. Cornblath EJ, Ashourvan A, Kim JZ, Betzel RF, Ciric R, Adebimpe A, et al. (2020): Temporal sequences of brain activity at rest are constrained by white matter structure and modulated by cognitive demands [no. 1]. Commun Biol 3: 1-12.

31. Gu S, Pasqualetti F, Cieslak M, Telesford QK, Yu AB, Kahn AE, et al. (2015): Controllability of structural brain networks. Nat Commun 6: 1-10.

32. Betzel RF, Gu S, Medaglia JD, Pasqualetti F, Bassett DS (2016): Optimally controlling the human connectome: the role of network topology. Sci Rep 6: 30770.

33. Gu S, Betzel RF, Mattar MG, Cieslak M, Delio PR, Grafton ST, et al. (2017): Optimal trajectories of brain state transitions. Neurolmage 148: 305-317. 
34. Kringelbach ML, Cruzat J, Cabral J, Knudsen GM, Carhart-Harris R, Whybrow PC, et al. (2020): Dynamic coupling of whole-brain neuronal and neurotransmitter systems. Proc Natl Acad Sci 117: 9566-9576.

35. Daniel SE, Lees AJ (1993): Parkinson's Disease Society Brain Bank, London: overview and research. J Neural Transm Suppl 39: 165-172.

36. Papapetropoulos S, Katzen H, Schrag A, Singer C, Scanlon BK, Nation D, et al. (2008): A questionnaire-based (UM-PDHQ) study of hallucinations in Parkinson's disease. BMC Neurol 8: 21.

37. Zarkali A, McColgan P, Leyland L-A, Lees AJ, Rees G, Weil RS (2021): Organisational and neuromodulatory underpinnings of structural-functional connectivity decoupling in patients with Parkinson's disease [no. 1]. Commun Biol 4: 1-13.

38. Esteban O, Markiewicz CJ, Blair RW, Moodie CA, Isik Al, Erramuzpe A, et al. (2019):

fMRIPrep: a robust preprocessing pipeline for functional MRI. Nat Methods 16: 111116.

39. Behzadi Y, Restom K, Liau J, Liu TT (2007): A component based noise correction method (CompCor) for BOLD and perfusion based fMRI. Neurolmage 37: 90-101.

40. Saad ZS, Gotts SJ, Murphy K, Chen G, Jo HJ, Martin A, Cox RW (2012): Trouble at Rest: How Correlation Patterns and Group Differences Become Distorted After Global Signal Regression. Brain Connect 2: 25-32.

41. Tournier J-D, Smith R, Raffelt D, Tabbara R, Dhollander T, Pietsch M, et al. (2019):

MRtrix3: A fast, flexible and open software framework for medical image processing and visualisation. Neurolmage 202: 116137.

42. Veraart J, Fieremans E, Novikov DS (2016): Diffusion MRI noise mapping using random matrix theory. Magn Reson Med 76: 1582-1593. 
43. Kellner E, Dhital B, Kiselev VG, Reisert M (2016): Gibbs-ringing artifact removal based on local subvoxel-shifts. Magn Reson Med 76: 1574-1581.

44. Anderson G (2006): Assuring Quality/Resisting Quality Assurance: Academics' responses to 'quality' in some Australian universities. Qual High Educ 12: 161-173.

45. Tustison NJ, Avants BB, Cook PA, Zheng Y, Egan A, Yushkevich PA, Gee JC (2010): N4ITK: improved N3 bias correction. IEEE Trans Med Imaging 29: 1310-1320.

46. Schaefer A, Kong R, Gordon EM, Laumann TO, Zuo X-N, Holmes AJ, et al. (2018): LocalGlobal Parcellation of the Human Cerebral Cortex from Intrinsic Functional Connectivity MRI. Cereb Cortex N Y N 1991 28: 3095-3114.

47. Tian Y, Margulies DS, Breakspear M, Zalesky A (2020): Topographic organization of the human subcortex unveiled with functional connectivity gradients [no. 11]. Nat Neurosci 23: 1421-1432.

48. Luppi Al, Stamatakis EA (2021): Combining network topology and information theory to construct representative brain networks. Netw Neurosci 5: 96-124.

49. Messé $A$ (2020): Parcellation influence on the connectivity-based structure-function relationship in the human brain. Hum Brain Mapp 41: 1167-1180.

50. Preti MG, Bolton TA, Van De Ville D (2017): The dynamic functional connectome: Stateof-the-art and perspectives. Neurolmage 160: 41-54.

51. Fukushima M, Betzel RF, He Y, van den Heuvel MP, Zuo X-N, Sporns O (2018): Structurefunction relationships during segregated and integrated network states of human brain functional connectivity. Brain Struct Funct 223: 1091-1106.

52. Blondel VD, Guillaume J-L, Lambiotte R, Lefebvre E (2008): Fast unfolding of communities in large networks. J Stat Mech Theory Exp 2008: P10008. 
53. Hollander, T., Raffelt D, Connelly A (2016): Unsupervised 3-tissue response function estimation from single-shell or multi-shell diffusion MR data without a co-registered T1 image. ISMRM Workshop Break Barriers Diffus MRI 5.

54. Smith RE, Tournier J-D, Calamante F, Connelly A (2012): Anatomically-constrained tractography: improved diffusion MRI streamlines tractography through effective use of anatomical information. Neurolmage 62: 1924-1938.

55. Smith RE, Tournier J-D, Calamante F, Connelly A (2015): SIFT2: Enabling dense quantitative assessment of brain white matter connectivity using streamlines tractography. Neurolmage 119: 338-351.

56. Kim JZ, Soffer JM, Kahn AE, Vettel JM, Pasqualetti F, Bassett DS (2018): Role of graph architecture in controlling dynamical networks with applications to neural systems [no. 1]. Nat Phys 14: 91-98.

57. Zöller D, Sandini C, Schaer M, Eliez S, Bassett DS, Ville DVD (2021): Structural control energy of resting-state functional brain states reveals less cost-effective brain dynamics in psychosis vulnerability. Hum Brain Mapp 42: 2181-2200.

58. Cui Z, Stiso J, Baum GL, Kim JZ, Roalf DR, Betzel RF, et al. (2020): Optimization of energy state transition trajectory supports the development of executive function during youth ((T. Yeo \& T. E. Behrens, editors)). eLife 9: e53060.

59. Zalesky A, Fornito A, Bullmore ET (2010): Network-based statistic: Identifying differences in brain networks. Neurolmage 53: 1197-1207.

60. Dukart J, Holiga S, Rullmann M, Lanzenberger R, Hawkins PCT, Mehta MA, et al. (2021): JuSpace: A tool for spatial correlation analyses of magnetic resonance imaging data with nuclear imaging derived neurotransmitter maps. Hum Brain Mapp 42: 555-566. 
61. Hawrylycz M, Miller JA, Menon V, Feng D, Dolbeare T, Guillozet-Bongaarts AL, et al.

(2015): Canonical genetic signatures of the adult human brain. Nat Neurosci 18:

1832-1844.

62. Alexander-Bloch A, Shou H, Liu S, Satterthwaite TD, Glahn DC, Shinohara RT, et al.

(2018): On testing for spatial correspondence between maps of human brain

structure and function. Neurolmage 178: 540-551.

63. Váša F, Seidlitz J, Romero-Garcia R, Whitaker KJ, Rosenthal G, Vértes PE, et al. (2018):

Adolescent Tuning of Association Cortex in Human Structural Brain Networks. Cereb Cortex NY NY 28: 281-294.

64. Braun U, Harneit A, Pergola G, Menara T, Schaefer A, Betzel RF, et al. (2019): Brain state stability during working memory is explained by network control theory, modulated by dopamine D1/D2 receptor function, and diminished in schizophrenia. bioRxiv 679670.

65. Onofrj M, Espay AJ, Bonanni L, Pizzi SD, Sensi SL (2019): Hallucinations, somaticfunctional disorders of PD-DLB as expressions of thalamic dysfunction. Mov Disord 34: 1100-1111.

66. Aarsland D, Brønnick K, Ehrt U, De Deyn PP, Tekin S, Emre M, Cummings JL (2007):

Neuropsychiatric symptoms in patients with Parkinson's disease and dementia: frequency, profile and associated care giver stress. J Neurol Neurosurg Psychiatry 78: $36-42$.

67. Damaraju E, Allen EA, Belger A, Ford JM, McEwen S, Mathalon DH, et al. (2014): Dynamic functional connectivity analysis reveals transient states of dysconnectivity in schizophrenia. Neurolmage Clin 5: 298-308. 
68. Weber S, Johnsen E, Kroken RA, Løberg E-M, Kandilarova S, Stoyanov D, et al. (2020):

Dynamic Functional Connectivity Patterns in Schizophrenia and the Relationship

With Hallucinations. Front Psychiatry 11. https://doi.org/10.3389/fpsyt.2020.00227

69. Deco G, Cruzat J, Cabral J, Knudsen GM, Carhart-Harris RL, Whybrow PC, et al. (2018):

Whole-Brain Multimodal Neuroimaging Model Using Serotonin Receptor Maps

Explains Non-linear Functional Effects of LSD. Curr Biol CB 28: 3065-3074.e6.

70. Carhart-Harris RL, Muthukumaraswamy S, Roseman L, Kaelen M, Droog W, Murphy K, et

al. (2016): Neural correlates of the LSD experience revealed by multimodal

neuroimaging. Proc Natl Acad Sci 113: 4853-4858.

71. Weil RS, Hsu JK, Darby RR, Soussand L, Fox MD (2019): Neuroimaging in Parkinson's disease dementia: connecting the dots. Brain Commun 1.

https://doi.org/10.1093/braincomms/fcz006

72. Zarkali, Angeliki, McColgan, Peter, Leyland, Louise-Ann, Lees, Andrew, Weil, Rimona (2021): Longitudinal thalamic white and gray matter changes associated with visual hallucinations in Parkinson's disease.

73. Russo M, Carrarini C, Dono F, Rispoli MG, Di Pietro M, Di Stefano V, et al. (2019): The Pharmacology of Visual Hallucinations in Synucleinopathies. Front Pharmacol 10. https://doi.org/10.3389/fphar.2019.01379

74. Geyer MA, Vollenweider FX (2008): Serotonin research: contributions to understanding psychoses. Trends Pharmacol Sci 29: 445-453.

75. Cheng AV, Ferrier IN, Morris CM, Jabeen S, Sahgal A, McKeith IG, et al. (1991): Cortical serotonin-S2 receptor binding in Lewy body dementia, Alzheimer's and Parkinson's diseases. J Neurol Sci 106: 50-55. 
76. Huot P, Johnston TH, Darr T, Hazrati L-N, Visanji NP, Pires D, et al. (2010): Increased 5$\mathrm{HT} 2 \mathrm{~A}$ receptors in the temporal cortex of parkinsonian patients with visual hallucinations. Mov Disord Off J Mov Disord Soc 25: 1399-1408.

77. Cummings J, Isaacson S, Mills R, Williams H, Chi-Burris K, Corbett A, et al. (2014):

Pimavanserin for patients with Parkinson's disease psychosis: a randomised, placebo-controlled phase 3 trial. Lancet Lond Engl 383: 533-540.

78. University College, London (2019): Trial of Ondansetron as a Parkinson's HAllucinations Treatment. clinicaltrials.gov. Retrieved May 18, 2021, from https://clinicaltrials.gov/ct2/show/NCT04167813

79. Firbank MJ, Parikh J, Murphy N, Killen A, Allan CL, Collerton D, et al. (2018): Reduced occipital GABA in Parkinson disease with visual hallucinations. Neurology 91: e675e685.

80. Khundakar AA, Hanson PS, Erskine D, Lax NZ, Roscamp J, Karyka E, et al. (2016): Analysis of primary visual cortex in dementia with Lewy bodies indicates GABAergic involvement associated with recurrent complex visual hallucinations. Acta Neuropathol Commun 4: 66.

81. Iwaki H, Blauwendraat C, Leonard HL, Kim JJ, Liu G, Maple-Grødem J, et al. (2019): Genomewide association study of Parkinson's disease clinical biomarkers in 12 longitudinal patients' cohorts. Mov Disord Off J Mov Disord Soc 34: 1839-1850.

82. O'Callaghan C, Hezemans FH, Ye R, Rua C, Jones PS, Murley AG, et al. (2021): Locus coeruleus integrity and the effect of atomoxetine on response inhibition in Parkinson's disease. Brain. https://doi.org/10.1093/brain/awab142 
83. Vazey $\mathrm{E}$, Aston-Jones $\mathrm{G}$ (2012): The emerging role of norepinephrine in cognitive dysfunctions of Parkinson's disease. Front Behav Neurosci 6. https://doi.org/10.3389/fnbeh.2012.00048

84. Dahl MJ, Mather M, Sander MC, Werkle-Bergner M (2020): Noradrenergic Responsiveness Supports Selective Attention across the Adult Lifespan. J Neurosci 40: 4372-4390.

85. Jacob SN, Nienborg H (2018): Monoaminergic Neuromodulation of Sensory Processing. Front Neural Circuits 12. https://doi.org/10.3389/fncir.2018.00051

86. Power JD, Mitra A, Laumann TO, Snyder AZ, Schlaggar BL, Petersen SE (2014): Methods to detect, characterize, and remove motion artifact in resting state fMRI.

Neurolmage 84: 320-341.

87. Power JD, Laumann TO, Plitt M, Martin A, Petersen SE (2017): On Global fMRI Signals and Simulations. Trends Cogn Sci 21: 911-913.

88. Orban C, Kong R, Li J, Chee MWL, Yeo BTT (2020): Time of day is associated with paradoxical reductions in global signal fluctuation and functional connectivity. PLOS Biol 18: e3000602.

89. Honey CJ, Sporns O, Cammoun L, Gigandet X, Thiran JP, Meuli R, Hagmann P (2009): Predicting human resting-state functional connectivity from structural connectivity. Proc Natl Acad Sci U S A 106: 2035-2040.

90. Nozari E, Stiso J, Caciagli L, Cornblath EJ, He X, Bertolero MA, et al. (2020): Is the brain macroscopically linear? A system identification of resting state dynamics. bioRxiv 2020.12.21.423856. 
91. Schulz M-A, Yeo BTT, Vogelstein JT, Mourao-Miranada J, Kather JN, Kording K, et al. (2020): Different scaling of linear models and deep learning in UKBiobank brain images versus machine-learning datasets. Nat Commun 11: 4238.

92. Luppi Al, Mediano PAM, Rosas FE, Allanson J, Pickard JD, Williams GB, et al. (2021):

Paths to Oblivion: Common Neural Mechanisms of Anaesthesia and Disorders of Consciousness. bioRxiv 2021.02.14.431140. 